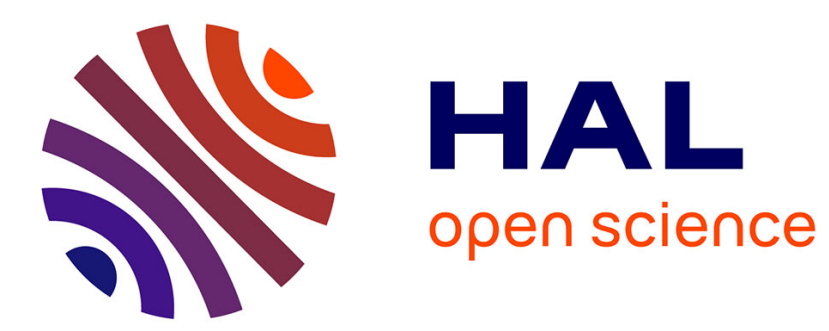

\title{
Gurus and beliefs manipulation
}

Elyès Jouini, Clotilde Napp

\section{- To cite this version:}

Elyès Jouini, Clotilde Napp. Gurus and beliefs manipulation. 2010. halshs-00555609

\section{HAL Id: halshs-00555609 \\ https://shs.hal.science/halshs-00555609}

Preprint submitted on 14 Jan 2011

HAL is a multi-disciplinary open access archive for the deposit and dissemination of scientific research documents, whether they are published or not. The documents may come from teaching and research institutions in France or abroad, or from public or private research centers.
L'archive ouverte pluridisciplinaire HAL, est destinée au dépôt et à la diffusion de documents scientifiques de niveau recherche, publiés ou non, émanant des établissements d'enseignement et de recherche français ou étrangers, des laboratoires publics ou privés. 


\section{Gurus and beliefs manipulation}

Elyès Jouini,

CEREMADE, Université Paris Dauphine

jouini@ceremade.dauphine.fr

+ 33144054226

Clotilde Napp,

DRM-Université Paris Dauphine and CNRS

clotilde.napp@dauphine.fr

+ 33144054642

December 2, 2010 


\title{
Gurus and beliefs manipulation
}

\begin{abstract}
We analyze a model with two types of agents: standard agents and gurus, i.e. agents who have the ability to influence the other investors. Gurus announce their beliefs and act accordingly. Each investor has a preferred guru and follows his recommendations. Prices are determined through a classical Walras mechanism. Gurus are strategic: they take into account the impact of their announced beliefs on the other agents, hence on prices. At the Nash equilibrium, this leads to beliefs heterogeneity, to a positive correlation between optimism and risk aversion and to higher risk premia. The impact is stronger on the riskier assets.
\end{abstract}




\section{Introduction}

In the classical financial economics theory, decision makers are assumed to have homogenous and rational expectations. This assumption has been the basis for many developments in finance. Among these developments, the portfolio selection model (Markowitz, 1952) and the Capital Asset Pricing Model (CAPM, Sharpe 1964 and Lintner 1965) play an important role. Given their simplicity and empirical tractability, these models and their subsequent extensions have become a significant cornerstone of theoretical and applied economics from insurance and financial theory to the theory of the firm.

However, the last 30 years have seen an accumulation of empirical tests that invalidate the theoretical conclusions of these models based on the assumption of homogeneous and objective beliefs. Furthermore, it suffices to observe the heterogeneity of analysts or professional forecasters forecasts or more generally of experts opinions to realize that this assumption is not realistic. In this paper, our aim is to analyze to what extent this heterogeneity may be generated by the presence of gurus that act strategically. We consider a model with two types of agents: "standard" investors and gurus (influential investors, newsletters writers,...). Like in Benabou-Laroque (2001), the "guru" we have in mind "issues forecasts but is also in the business of trading, for his own account or some investment firm." In our model, gurus announce their beliefs and each investor adopts the announced belief of one guru. This corresponds to the findings of Fisher-Statman (2000) where it appears that "there is a positive relationship between changes in the sentiment of individual investors and that of newsletter writers". The gurus choose their "beliefs" strategically taking into account the impact of their announced beliefs on other agents hence on prices.

The following example quoted by Benabou-Laroque (1992) "provides the most dramatic illustration of prices reacting to someone's announcement".

In the nervous market of 1987, Mr Prechter has emerged as both prophet and deity, an adviser whose advice reaches so many investors that he tends to pull the market the way he has predicted it will move [International Herald Tribune, October 3, $1987]$

For credibility reasons gurus act according to their announced beliefs. These strategic beliefs are not true beliefs from the gurus point of view, however they correspond to what the investors truly believe, moreover they are interpreted as gurus' beliefs by econometricians who observe 
portfolio choices.

We tackle the following issues. Do gurus have incentives to announce beliefs other than their true beliefs? How are investors' beliefs affected by the strategic interaction between gurus? Do the resulting beliefs exhibit optimism? pessimism? How are these possible biases related to the agents preferences? What is the impact of these beliefs on individual decisions? What is the impact of these beliefs on equilibrium characteristics such as prices and risk premium?

Our findings are the following. A strategic behavior leads to beliefs subjectivity and heterogeneity among the gurus even when gurus' initial beliefs coincide with the objective belief. Optimism as well as pessimism can both emerge. The intuition is as follows. Consider a model with two gurus. For the more risk tolerant guru, his demand in the risky asset is positive, so that his expected utility from trade is decreasing in the price of the risky asset. The choice of a pessimistic belief is associated with a lower demand, hence to a lower price, and the optimal belief balances this benefit of pessimism against the costs of worse decision making. The converse reasoning applies to the risk averse guru, who, at the equilibrium, has a negative demand in the risky asset and benefits from optimism. Depending on their levels of risk aversion, the agents adopt the beliefs of one of the gurus and we find a positive correlation across the agents between pessimism and risk tolerance.

Second, the representative agent belief, or the consensus belief, which is given by the average of the individual beliefs weighted by the risk tolerance, is pessimistic. Intuitively, the more risk tolerant agents make the market, and the consensus belief reflects the characteristics of the more risk tolerant. Since we have just seen that the more risk tolerant are pessimistic, it is consistent to obtain a pessimistic consensus belief. Such a pessimistic bias is also obtained in empirical studies in a purely behavioral setting (Ben Mansour et al., 2006), in a decision theory framework (Wakker, 2001) or in a market framework (Giordani-Söderlind, 2006). In particular, as underlined by Shefrin (2005) based on Wall \$treet Week data "between 1983 and 2002, professional investors were unduly pessimistic, underestimating market returns".

As a consequence of the pessimistic bias at the aggregate level, the risk premium is greater than in the standard rational expectations equilibrium. The fact that a pessimistic bias and a positive correlation between risk tolerance and pessimism lead to an increase of the market price of risk has been underlined by Abel (1989), Calvet et al. (2002), Detemple-Murthy (1994), Gollier (2007) and Jouini-Napp (2006); in their models, beliefs are exogenously given. This increase of the risk premium is interesting in light of the risk-premium puzzle on financial 
markets.

The paper is organized as follows. Section 2 presents the main model and the results. Section 3 provides three main extensions (considering private information, more than 2 assets, more than 2 gurus). Section 4 concludes. All proofs are provided in the Appendix.

\section{The model and the results}

We consider a model with a continuum of agents. Among these agents, there are 2 influential investors who have a wide audience. We do not assume that they have the capacity to move the markets by their own trades but only that they have, through their wide audience, the ability to largely influence the other investors. We will call them gurus and denote them by Guru $j$, $j=1,2$.

Gurus announce their beliefs and act accordingly in order to maintain their credibility. The announced beliefs are based on strategic considerations ${ }^{1}$.

The other agents in the economy believe that gurus are well informed or have specific ability to predict market movements. At a given date each agent has a preferred guru and adopts his beliefs. There are then 2 groups of agents: the agents in Group $j\left(G_{j}\right)$ follow Guru $j, j=1,2$.

\subsection{The gurus' game}

We focus on a single period and consumption takes place at the end of the period. There is a single risky asset in the economy, whose payoff at the end of the period is denoted by $\widetilde{x}$. We let $p$ denote the unit price of the risky asset, which means that agents can sell their property rights on the risky asset against the delivery of the sure quantity $p$ at the end of the period. We assume that there is one unit of the risky asset in the economy and that it is uniformly distributed among the agents. As in the standard portfolio problem, agents determine the optimal composition of their portfolio, in other words their optimal exposure to the risk. The difference with the standard model stems from the fact that agents adopt the beliefs of one of the two gurus and that the gurus take into account their impact as well as the impact of their followers on prices and can manipulate their beliefs to take advantage of this impact. For example, a guru, who is risk tolerant, hence willing to be quite highly exposed to the risk, or equivalently interested in buying a high quantity of the risky asset, could announce a more

\footnotetext{
${ }^{1}$ We shall see in Section 3 that the announced beliefs may also be based on private information.
} 
pessimistic belief and act as if he believed that the asset was less interesting (or as if he were less interested in buying the asset) in order to benefit from a lower price.

We assume that agents have CARA utility functions for consumption, more precisely, $u_{i}(c)=-\exp \left(-\frac{c}{\theta_{i}}\right)$ where $\theta_{i}>0$ denotes the degree of risk tolerance of agent $i$ for $i \in[0,1]$ and where $i \rightarrow \theta_{i}$ is measurable. We denote by $\Theta_{1}=\int_{G_{1}} \theta_{i} d i$ and by $\Theta_{2}=\int_{G_{2}} \theta_{i} d i$ the aggregate risk tolerance in $G_{1}$ and $G_{2}$. We have $\Theta_{1}+\Theta_{2}=\Theta$ where $\Theta$ is the aggregate risk tolerance in the economy ${ }^{2}$. Moreover, we assume that $\widetilde{x}$ is normally distributed, with mean $\mu$ and variance $\sigma^{2}$.

We assume that the gurus also have CARA utility functions, $u_{j}^{*}(c)=-\exp \left(-\frac{c}{\theta_{j}^{*}}\right)$ where $\theta_{j}^{*}>0$ denotes the degree of risk tolerance of Guru $j$ for $j=1,2$. Both gurus observe $\mu$. However, Guru $j$ announces a belief $\mu_{j}$ and this belief is adopted by all the agents in $G_{j}$. The belief $\mu_{j}$ is then chosen strategically. For credibility reasons, gurus act according to their announced beliefs. A possible justification for such a behavior is that agents can observe gurus' portfolios and may eliminate a guru whose portfolio choices are not consistent with his announced forecasts.

As already noticed, the announced belief is not a true belief for the guru but it will be considered as such by an observer/econometrician who analyzes gurus portfolio choices. It is also a true belief for the other agents since they sincerely trust one of the gurus and adopt his announced belief as their own true belief.

For given announced beliefs $\left(\mu_{1}, \mu_{2}\right)$, the optimal demand $\alpha_{i}(p)$ of the risky asset that agent $i$ will retain given price $p$ maximizes the expected utility from trade

$$
E^{i}\left[-\exp -\frac{p+\alpha_{i}(\widetilde{x}-p)}{\theta_{i}}\right]
$$

where $E^{i}$ corresponds to the expectation operator associated to agent $i$ belief. If agent $i$ adopts the belief of Guru $j$ then the optimal demand is given by $\alpha_{i}(p)=\theta_{i} \frac{\mu_{j}-p}{\sigma^{2}}$. The total demand of Group $j$ is given by

$$
\alpha_{G_{j}}(p)=\Theta_{j} \frac{\mu_{j}-p}{\sigma^{2}}
$$

and corresponds to the demand of a representative agent for Group $j$ with belief $\mu_{j}$ and risk tolerance $\Theta_{j}$. As underlined, all the agents, including the Gurus, are infinitesimal and the

\footnotetext{
${ }^{2} \mathrm{~A}$ model with a continuum of agents permits to approximate models with a large number of agents. Furthermore, all pairs $\left(\Theta_{1}, \Theta_{2}\right)$ with $\Theta_{1}+\Theta_{2}=\Theta$ are possible in such a model. The analysis would be essentially the same in a model with a finite number of agents except that we should choose the $\Theta_{i}^{\prime}$ s on a grid.
} 
individual demands also are infinitesimal. For a given price, the total demand of risky asset in the economy is then the sum of the total demands of Group 1 and of Group 2. The equilibrium price $p\left(\mu_{1}, \mu_{2}\right)$ is then determined by the condition

$$
\Theta_{1} \frac{\mu_{1}-p}{\sigma^{2}}+\Theta_{2} \frac{\mu_{2}-p}{\sigma^{2}}=1
$$

and given by

$$
p\left(\mu_{1}, \mu_{2}\right)=\frac{\Theta_{1} \mu_{1}+\Theta_{2} \mu_{2}}{\Theta}-\frac{\sigma^{2}}{\Theta} .
$$

Moreover, replacing $p$ by its expression in $\alpha_{G_{j}}(p)$ we obtain that the aggregate optimal demand at the equilibrium for Group 1 (Group 2) are given by

$$
\begin{aligned}
& \alpha_{G_{1}}=\frac{\Theta_{1}}{\Theta}\left[1+\Theta_{2} \frac{\mu_{1}-\mu_{2}}{\sigma^{2}}\right] \\
& \alpha_{G_{2}}=\frac{\Theta_{2}}{\Theta}\left[1+\Theta_{1} \frac{\mu_{2}-\mu_{1}}{\sigma^{2}}\right]
\end{aligned}
$$

and the part of the risk borne by Group $j$ depends upon both his level of risk tolerance and his belief. Letting $R P$ (resp. $R P^{s t d d}$ ) denote the risk premium $\mu-p$ in this setting (resp. in the standard setting where all beliefs coincide with the objective belief $\mu$ ) we obtain

$$
R P\left(\mu_{1}, \mu_{2}, \Theta_{1}, \Theta_{2}\right)=\frac{\sigma^{2}}{\Theta}+\left(\mu-\frac{\Theta_{1} \mu_{1}+\Theta_{2} \mu_{2}}{\Theta}\right)=R P^{s t d d}+\left(\mu-\frac{\Theta_{1} \mu_{1}+\Theta_{2} \mu_{2}}{\Theta}\right)
$$

which means that the risk premium in an economy with heterogeneous subjective beliefs is higher than in the standard rational expectations setting if and only if the belief of the representative agent, which is the risk tolerance weighted average of the individual beliefs, is pessimistic, where pessimistic is meant in the sense that the mean of the risky asset's payoff is underestimated. In such a setting, it is particularly interesting to explore when and why the representative agent is pessimistic. In the present paper, the individual beliefs result from the gurus beliefs which are determined endogenously. We shall then analyze their properties, especially in terms of pessimism, correlation between pessimism and risk tolerance and impact on the risk premium.

The demand of Guru $j$ is determined, as usual, by his own level of risk tolerance $\theta_{j}^{*}$ and by his announced belief $\mu_{j}$. Recall that gurus act according to their announced beliefs in order to 
maintain their credibility. The demand of Guru $j$ is given by

$$
\alpha_{j}^{*}\left(p, \mu_{j}\right)=\theta_{j}^{*} \frac{\mu_{j}-p}{\sigma^{2}} .
$$

The utility level of Guru $j$ is given by

$$
\begin{aligned}
U_{j}\left(\mu_{1}, \mu_{2}\right) & =E\left[u_{j}^{*}\left(p\left(\mu_{1}, \mu_{2}\right)+\left(\tilde{x}-p\left(\mu_{1}, \mu_{2}\right)\right) \alpha_{j}^{*}\left(p\left(\mu_{1}, \mu_{2}\right), \mu_{j}\right)\right)\right] \\
& =E\left[-\exp \left(-\frac{1}{\theta_{j}^{*}}\left(p\left(\mu_{1}, \mu_{2}\right)+\left(\tilde{x}-p\left(\mu_{1}, \mu_{2}\right)\right) \alpha_{j}^{*}\left(p\left(\mu_{1}, \mu_{2}\right), \mu_{j}\right)\right)\right)\right] .
\end{aligned}
$$

Guru $j$ acts strategically and chooses $\widehat{\mu}_{j}$ that maximizes his utility level

$$
U_{j}\left(\widehat{\mu}_{i}, \mu_{j}\right)=E\left[-\exp \left(-\frac{1}{\theta_{j}^{*}}\left(p\left(\widehat{\mu}_{i}, \mu_{j}\right)+\left(\tilde{x}-p\left(\widehat{\mu}_{i}, \mu_{j}\right)\right) \alpha_{j}^{*}\left(p\left(\widehat{\mu}_{i}, \mu_{j}\right), \mu_{j}\right)\right)\right)\right]
$$

where $\widehat{\mu}_{i}$ denotes the strategy of Guru $i$ and is taken as given. We emphasize that the choice of the belief $\widehat{\mu_{j}}$ is strategic: the guru believes that the mean of $\widetilde{x}$ is $\mu$ but he behaves as if he truly believed that it is $\widehat{\mu_{j}}$ in order to take advantage of his impact (through his influence on other agents beliefs) on prices and to maximize his utility from profits.

The gurus then play a game defined by payoff functions $U_{j}, j=1,2$ and their strategic variable is their belief $\mu_{j}$. The parameters of this game are $\left(\Theta_{1}, \Theta_{2}\right)$ and we denote it by $\Gamma\left(\Theta_{1}, \Theta_{2}\right)$.

Definition 1 An equilibrium with strategic beliefs manipulation is a Nash equilibrium of the game $\Gamma\left(\Theta_{1}, \Theta_{2}\right)$, i.e. a pair of strategies $M=\left(\widehat{\mu}_{1}\left(\Theta_{1}, \Theta_{2}\right), \widehat{\mu}_{2}\left(\Theta_{1}, \Theta_{2}\right)\right)$ for the gurus such that for any other pair of strategies $M^{\prime}$ differing only in the $j$-th component, for $j=1,2$, the strategy $M$ yields a utility level no less than $M^{\prime}$ :

$$
E\left[u_{j}^{*}\left(p(M)+(\widetilde{x}-p(M)) \alpha_{j}^{*}(M)\right)\right] \geq E\left[u_{j}^{*}\left(p\left(M^{\prime}\right)+\left(\widetilde{x}-p\left(M^{\prime}\right)\right) \alpha_{j}^{*}\left(M^{\prime}\right)\right)\right] .
$$

Note that the risk tolerance levels involved in the price formula (1) are not those of the gurus but those of their respective groups. In particular, we may have $\theta_{1}^{*}>\theta_{2}^{*}$ with $\Theta_{1}<\Theta_{2}$, i.e. the more risk tolerant guru may have a lower weight in the price formula than the other guru (or equivalently the risk tolerance level of his group is lower than the risk tolerance level of the other group). The payoff function then involves two different levels of risk tolerance, the individual level of risk tolerance of the guru under consideration through the form of the 
utility function and the level of risk tolerance of the group associated with that guru through the price formula. Our game is then different from an imperfect competition game where each agent would take into account his own direct impact on equilibrium prices in order to determine his optimal strategy (as in e.g. Jouini et al., 2010).

Proposition 1 If the weight (or the risk tolerance level) of Group $i$ is given by $\Theta_{i}$, the model admits a unique equilibrium with strategic beliefs manipulation, i.e. the game $\Gamma\left(\Theta_{1}, \Theta_{2}\right)$ has a unique Nash equilibrium $\left(\widehat{\mu}_{1}\left(\Theta_{1}, \Theta_{2}\right), \widehat{\mu}_{2}\left(\Theta_{1}, \Theta_{2}\right)\right)$ with

$$
\begin{aligned}
& \widehat{\mu}_{1}=\mu+\frac{1}{2} \frac{\left(\theta_{1}^{*} \Theta_{2}^{2}+\theta_{2}^{*} \Theta_{1}^{2}+2 \theta_{2}^{*} \Theta_{1} \Theta_{2}-\theta_{1}^{*} \theta_{2}^{*} \Theta\right)}{\Theta \theta_{1}^{*} \theta_{2}^{*} \Theta_{2}} \sigma^{2}, \\
& \widehat{\mu}_{2}=\mu+\frac{1}{2} \frac{\left(\theta_{1}^{*} \Theta_{2}^{2}+\theta_{2}^{*} \Theta_{1}^{2}+2 \theta_{1}^{*} \Theta_{1} \Theta_{2}-\theta_{1}^{*} \theta_{2}^{*} \Theta\right)}{\Theta \theta_{1}^{*} \theta_{2}^{*} \Theta_{1}} \sigma^{2} .
\end{aligned}
$$

\subsection{The groups' dynamics}

We have so far considered the parameters $\Theta_{i}=\int_{G_{i}} \theta_{i} d i, i=1,2$, as given. Let us now analyze how agents may choose their respective groups. We may imagine that the agents play several times the same game with possibly different values for $\mu$ at each step. After each step, the agents observe market realizations and compare them with gurus' predictions. They evaluate the performance of each guru and their probability of choosing a given guru for the next step is then related to the latter's success ${ }^{3}$. More generally, the performance evaluation of each guru by a given agent may be based on agent's own experience with this guru. In particular, agents may move from one guru to another based upon their initial priors, their own experience, their past choices and the observed realizations. In such a setting the choice of a guru is an individual decision and the switches from one guru to another do not necessarily occur at the same time for all agents. We may also imagine that gurus are ranked by independent agencies on the basis of the accuracy of their predictions and that the agents base their choice of a specific guru upon these rankings.

Whatever the dynamics is, the steady states should respect the following natural condition $\left|\widehat{\mu}_{i}\left(\Theta_{1}, \Theta_{2}\right)-\mu\right|=\left|\widehat{\mu}_{j}\left(\Theta_{1}, \Theta_{2}\right)-\mu\right|$ that reflects the fact that in the long run gurus' predictions should have the same level of accuracy. Let us denote by $\mathbb{D}=\left\{\left(\Theta_{1}, \Theta_{2}\right) \in \mathbb{R}_{+} \times \mathbb{R}_{+}: \Theta_{1}+\Theta_{2}=\Theta\right\}$.

\footnotetext{
${ }^{3}$ As in Föllmer et al. (2004) the agents learn on gurus' ability and construct choice functions based on this learning. There is a substantial literature on such adaptative learning rules which are associated with "discrete choice" models; see e.g., Anderson et al. (1992). Different forms of these rules have been used and accounts are given in Weisbuch et al. (1998) and Brock and Durlauf (2001a, 2001b).
} 
Definition An equilibrium for the groups' dynamics is an element $\left(\Theta_{1}^{*}, \Theta_{2}^{*}\right)$ of $\mathbb{D}$ such that $\left|\widehat{\mu}_{1}\left(\Theta_{1}^{*}, \Theta_{2}^{*}\right)-\mu\right|=\left|\widehat{\mu}_{2}\left(\Theta_{1}^{*}, \Theta_{2}^{*}\right)-\mu\right|$. Such an equilibrium $\left(\Theta_{1}^{*}, \Theta_{2}^{*}\right)$ is said to be stable if, in a neighborhood of $\left(\Theta_{1}^{*}, \Theta_{2}^{*}\right)$, we have $\left|\widehat{\mu}_{1}\left(\Theta_{1}, \Theta_{2}\right)-\mu\right|<\left|\widehat{\mu}_{2}\left(\Theta_{1}, \Theta_{2}\right)-\mu\right|$ for $\Theta_{1}<\Theta_{1}^{*}$ and $\left|\widehat{\mu}_{1}\left(\Theta_{1}, \Theta_{2}\right)-\mu\right|>\left|\widehat{\mu}_{2}\left(\Theta_{1}, \Theta_{2}\right)-\mu\right|$ for $\Theta_{1}>\Theta_{1}^{*}$. It is said to be globally stable if this property is satisfied on the whole domain $\mathbb{D}$.

The stability concept introduced in the previous definition is fairly natural. When the size of one of the two groups, let us say Group 1, is slightly modified towards an increase, the relative accuracy of Guru 2 (resp. 1) increases leading to a migration of agents from Group 1 to Group 2 .

Proposition 2 Let us assume that one guru is more risk tolerant than the average of the agents while the other guru is less risk tolerant than the average, i.e. $\theta_{1}^{*}>\Theta>\theta_{2}^{*}$. Then

1. There exists a unique equilibrium $\left(\Theta_{1}^{*}, \Theta_{2}^{*}\right)$ for the groups' dynamics. It is given as the unique solution $\Theta_{1}^{*}$ of

$$
\Theta_{1}^{3}\left(\theta_{1}^{*}-\theta_{2}^{*}\right)-3 \Theta \Theta_{1}^{2}\left(\theta_{1}^{*}-\theta_{2}^{*}\right)+\Theta^{2} \theta_{1}^{*}\left(\Theta-\theta_{2}^{*}\right)=0
$$

2. The equilibrium $\left(\Theta_{1}^{*}, \Theta_{2}^{*}\right)$ is globally stable.

3. At the equilibrium $\left(\Theta_{1}^{*}, \Theta_{2}^{*}\right)$, the utility level of a given agent is not affected by the choice of a specific guru.

4. If the gurus are on average as risk tolerant as the whole population, i.e. $\Theta=\frac{\theta_{1}^{*}+\theta_{2}^{*}}{2}$, then $\Theta_{1}^{*}>\Theta_{2}^{*}$, the representative agent belief is pessimistic, i.e. $\frac{\Theta_{1}^{*} \widehat{\mu}_{1}\left(\Theta_{1}^{*}, \Theta_{2}^{*}\right)+\Theta_{2}^{*} \widehat{\mu}_{2}\left(\Theta_{1}^{*}, \Theta_{2}^{*}\right)}{\Theta}<\mu$, and the risk premium is increased, i.e. $R P\left(\widehat{\mu}_{1}\left(\Theta_{1}^{*}, \Theta_{2}^{*}\right), \widehat{\mu}_{2}\left(\Theta_{1}^{*}, \Theta_{2}^{*}\right), \Theta_{1}^{*}, \Theta_{2}^{*}\right)>R P^{s t d d}$.

5. If $\Theta=\frac{\theta_{1}^{*}+\theta_{2}^{*}}{2}$ and if the two groups have the same number of agents, i.e. $\int_{G_{1}} d i=\int_{G_{2}} d i=$ $\frac{1}{2}$, then there is a positive correlation between pessimism and risk tolerance among the whole population.

The first assertion means that there is a unique distribution of risk tolerance among Group 1 and Group 2 for which the associated Nash equilibrium satisfies $\left|\widehat{\mu}_{1}-\mu\right|=\left|\widehat{\mu}_{2}-\mu\right|$ leading to the same level of accuracy for both gurus. The second assertion means that for any initial distribution of risk tolerance among the two groups, any dynamics that favors the most accurate guru converges to the distribution $\left(\Theta_{1}^{*}, \Theta_{2}^{*}\right)$. The third assertion provides an additional stability 
result. Our initial stability concept is only based on the level of accuracy of gurus' predictions. However, even if agents take their utility level into account in the choice of their guru, they will be indifferent between Guru 1 and Guru 2. The fourth assertion means that the more risk tolerant group is the group of the more risk tolerant guru and also the most pessimistic one when the gurus are on average as risk tolerant as the whole population. Since each group behaves like a representative agent whose risk tolerance is given by the total risk tolerance within the group it is natural to obtain that the more (less) risk tolerant group is in line with the more (less) risk tolerant guru. This in turn implies that the representative agent is pessimistic and the risk premium is increased. The condition introduced in 5. about the relative sizes of the groups is natural. Indeed, when agents are indifferent between Guru 1 and Guru 2 from both accuracy and utility point of views (as seen in 2. and 3.), there is no specific reason to choose one guru rather than the other one. If agents choose each of the two gurus with a probability $\frac{1}{2}$, then

the two groups are of equal size, i.e. $\int_{G_{1}} d i=\int_{G_{2}} d i=\frac{1}{2}$. We then obtain a positive correlation between pessimism and risk tolerance among the whole population as in Ben Mansour et al. (2006).

To conclude and roughly speaking, at a steady state and if the two gurus are on average as risk tolerant as the whole population, then the more risk tolerant guru is pessimistic, the less risk tolerant guru is optimistic and there is a positive correlation between pessimism and risk tolerance among the whole population. This implies that the strategic behavior leads to a higher risk premium.

\section{Extensions}

\subsection{The model with private information}

In the previous section, we assumed that the gurus observe the objective belief. Let us consider the situation where the two gurus have different initial beliefs. We assume that Guru $i$ has an initial belief $\nu_{i}$ and distorts it strategically. As previously, he acts as if he had a belief $\widehat{\mu}_{i}$ that is strategically determined by the maximization of the utility level

$$
U_{i}\left(\mu_{i}, \widehat{\mu_{j}}\right)=E^{i}\left[-\exp \left(-\frac{1}{\theta_{j}^{*}}\left(p\left(\mu_{i}, \widehat{\mu_{j}}\right)+\left(\tilde{x}-p\left(\mu_{i}, \widehat{\mu_{j}}\right)\right) \alpha_{i}^{*}\left(p\left(\mu_{i}, \widehat{\mu_{j}}\right), \mu_{i}\right)\right)\right)\right]
$$


where the equilibrium price $p$ and Guru $i$ 's demand $\alpha_{i}^{*}$ are given by Equations (2) and (4) and where $E^{i}$ is the expectation operator associated to the prior of Guru $i$. The gurus play then a game defined by payoff functions $U_{i}, i=1,2$ and their strategic variable is their belief $\mu_{i}$. The parameters of this game are $\left(\Theta_{1}, \Theta_{2}, \nu_{1}, \nu_{2}\right)$ and we denote it by $\Gamma\left(\Theta_{1}, \Theta_{2}, \nu_{1}, \nu_{2}\right)$.

Proposition 3 If Guru $i$ has an initial belief $\nu_{i}$ and Group $i$ has a weight (or a level of risk tolerance) $\Theta_{i}$ then the model admits a unique equilibrium with strategic beliefs manipulation, i.e. the game $\Gamma\left(\Theta_{1}, \Theta_{2}, \nu_{1}, \nu_{2}\right)$ has a unique Nash equilibrium $\left(\widehat{\mu}_{1}, \widehat{\mu}_{2}\right)$ with

$$
\begin{aligned}
\widehat{\mu_{1}} & =\frac{1}{2} \frac{\theta_{1} \theta_{2} \Theta_{1} \Theta_{2}\left(\nu_{1}+\nu_{2}\right)-\sigma^{2} \theta_{1} \theta_{2} \Theta+2 \sigma^{2} \theta_{2} \Theta_{1} \Theta_{2}+2 \theta_{1} \theta_{2} \Theta_{2}^{2} \nu_{1}+\sigma^{2} \theta_{1} \Theta_{2}^{2}+\sigma^{2} \theta_{2} \Theta_{1}^{2}}{\theta_{1} \theta_{2} \Theta_{2} \Theta} \\
\widehat{\mu_{2}} & =\frac{1}{2} \frac{\theta_{1} \theta_{2} \Theta_{1} \Theta_{2}\left(\nu_{1}+\nu_{2}\right)-\sigma^{2} \theta_{1} \theta_{2} \Theta+2 \sigma^{2} \theta_{1} \Theta_{1} \Theta_{2}+2 \theta_{1} \theta_{2} \Theta_{1}^{2} \nu_{2}+\sigma^{2} \theta_{1} \Theta_{2}^{2}+\sigma^{2} \theta_{2} \Theta_{1}^{2}}{\theta_{1} \theta_{2} \Theta_{1} \Theta},
\end{aligned}
$$

and the equilibrium price is given by

$$
p\left(\widehat{\mu_{1}}, \widehat{\mu_{2}}\right)=\frac{1}{2} \frac{\theta_{1} \theta_{2} \Theta_{1} \Theta_{2}\left(\nu_{1}+\nu_{2}\right)}{\theta_{1} \theta_{2} \Theta_{1} \Theta_{2}}-\frac{1}{2} \frac{\theta_{1} \Theta_{2}^{2}+\theta_{2} \Theta_{1}^{2}-\Theta \theta_{1} \theta_{2}}{\theta_{1} \theta_{2} \Theta_{1} \Theta_{2}} \sigma^{2}
$$

When the different initial beliefs correspond to differences of opinions, then there is no relevant informational content in the equilibrium price. However, if the different initial beliefs result from different signals received by the agents, then the equilibrium price transmits relevant information and the right concept to use is the concept of rational expectations equilibrium.

Let us assume that the agents have the same initial prior $\tilde{x} \sim \mathcal{N}\left(\mu, \sigma^{2}\right)$ and that Guru $i$, for $i=1,2$, observes a signal $\tilde{y}_{i}=\tilde{x}+\tilde{\varepsilon}_{i}$ where $\tilde{\varepsilon}_{1}$ and $\tilde{\varepsilon}_{2}$ are independent and follow the same distribution $\mathcal{N}\left(0, \sigma_{\varepsilon}^{2}\right)$. Guru $i$ 's belief after the observation of a given realization $y_{i}$ is then given by $\mathcal{N}\left(\nu_{i}, \check{\sigma}^{2}\right)$ with $\nu_{i}=\mu+\left(y_{i}-\mu\right) \frac{\sigma^{2}}{\sigma_{\varepsilon}^{2}+\sigma^{2}}$ and $\check{\sigma}^{2}=\frac{\sigma^{2} \sigma_{\varepsilon}^{2}}{\sigma_{\varepsilon}^{2}+\sigma^{2}}$.

In such a model with private information a rational expectations equilibrium with strategic beliefs manipulation is defined by a pair of announced beliefs $\left(\widehat{\mu}_{1}\left(y_{1}, y_{2}\right), \widehat{\mu}_{2}\left(y_{1}, y_{2}\right)\right)$ and by a price functional $P\left(y_{1}, y_{2}\right)$ such that $P\left(y_{1}, y_{2}\right)=\frac{\Theta_{1} \widehat{\mu}_{1}\left(y_{1}, y_{2}\right)+\Theta_{2} \widehat{\mu}_{2}\left(y_{1}, y_{2}\right)}{\Theta}-\frac{\sigma^{2}}{\Theta}$ (i.e. the markets clear when Group 1 follows the announced belief of Guru 1 and Group 2 follows the announced belief of Guru 2) and the belief $\widehat{\mu}_{i}\left(y_{1}, y_{2}\right)$ of Guru $i$ maximizes his expected utility

$$
U_{i}\left(\mu_{i}, \widehat{\mu_{j}}\right)=E\left[-\exp \left(-\frac{1}{\theta_{i}^{*}}\left(p\left(\mu_{i}, \widehat{\mu_{j}}\right)+\left(\tilde{x}-p\left(\mu_{i}, \widehat{\mu_{j}}\right)\right) \alpha_{i}^{*}\left(p\left(\mu_{i}, \widehat{\mu_{j}}\right), \mu_{i}\right)\right)\right) \mid y_{i}, P\left(y_{1}, y_{2}\right)\right]
$$


where the price functional $p\left(\mu_{i}, \mu_{j}\right)$ is defined by Equation ${ }^{4}(2)$, where the belief of Guru $j$ is considered as given and where the expectation is conditional to the private information of Guru $i$ and to the public information conveyed by prices.

Following Equation (8), when the agents only take into account their private information, the equilibrium price is given by

$$
p\left(\widehat{\mu_{1}}, \widehat{\mu_{2}}\right)=\frac{\theta_{1} \theta_{2} \Theta_{1} \Theta_{2}\left(\mu+(\bar{y}-\mu) \frac{\sigma^{2}}{\sigma_{\varepsilon}^{2}+\sigma^{2}}\right)}{\theta_{1} \theta_{2} \Theta_{1} \Theta_{2}}-\frac{1}{2} \frac{\theta_{1} \Theta_{2}^{2}+\theta_{2} \Theta_{1}^{2}-\Theta \theta_{1} \theta_{2}}{\theta_{1} \theta_{2} \Theta_{1} \Theta_{2}} \check{\sigma}^{2}
$$

where $\bar{y}=\frac{y_{1}+y_{2}}{2}$ is the average signal.

The equilibrium price is then fully revealing and each agent is able to infer the average signal from price observation. Since the average signal is a sufficient statistics of all the agents' available information, then the agents share the same belief about $\tilde{x}$ after the observation of the equilibrium price and it is given by $\mathcal{N}\left(\breve{\mu}, \breve{\sigma}^{2}\right)$ where $\breve{\mu}=\mu+(\bar{y}-\mu) \frac{\sigma^{2}}{\frac{1}{2} \sigma_{\varepsilon}^{2}+\sigma^{2}}$ and $\breve{\sigma}^{2}=\frac{\sigma^{2} \sigma_{\varepsilon}^{2}}{\sigma_{\varepsilon}^{2}+2 \sigma^{2}}$. Everything works as if both agents had observed a same signal $\bar{y}=\frac{y_{1}+y_{2}}{2}$ which variance is given by $\frac{\sigma_{\varepsilon}^{2}}{2}$. As usual, it is easy to check that the fully revealing rational expectations equilibrium corresponds to a standard equilibrium where the agents would share their information before trading. All the information contained in the price is then already used by the agents when they choose their strategic belief.

Proposition 4 If Guru $i$ receives a private signal $\tilde{y}_{i}=\tilde{x}+\tilde{\varepsilon}_{i}$ where $\tilde{\varepsilon}_{1}$ and $\tilde{\varepsilon}_{2}$ are independent and follow the same distribution $\mathcal{N}\left(0, \sigma_{\varepsilon}^{2}\right)$ and if Group $i$ has a weight $\Theta_{i}$ then $\left(\left(\widehat{\mu}_{1}\left(y_{1}, y_{2}\right), \widehat{\mu}_{2}\left(y_{1}, y_{2}\right), p\left(y_{1}, y_{2}\right)\right)\right.$ defined by

$$
\begin{aligned}
\widehat{\mu}_{1}\left(y_{1}, y_{2}\right) & =\mu+\left(\frac{y_{1}+y_{2}}{2}-\mu\right) \frac{\sigma^{2}}{\frac{1}{2} \sigma_{\varepsilon}^{2}+\sigma^{2}}+\frac{1}{2} \frac{\left(\theta_{1}^{*} \Theta_{2}^{2}+\theta_{2}^{*} \Theta_{1}^{2}+2 \theta_{2}^{*} \Theta_{1} \Theta_{2}-\theta_{1}^{*} \theta_{2}^{*} \Theta\right)}{\Theta \theta_{1}^{*} \theta_{2}^{*} \Theta_{2}} \frac{\sigma^{2} \sigma_{\varepsilon}^{2}}{\sigma_{\varepsilon}^{2}+2 \sigma^{2}}, \\
\widehat{\mu}_{2}\left(y_{1}, y_{2}\right) & =\mu+\left(\frac{y_{1}+y_{2}}{2}-\mu\right) \frac{\sigma^{2}}{\frac{1}{2} \sigma_{\varepsilon}^{2}+\sigma^{2}}+\frac{1}{2} \frac{\left(\theta_{1}^{*} \Theta_{2}^{2}+\theta_{2}^{*} \Theta_{1}^{2}+2 \theta_{1}^{*} \Theta_{1} \Theta_{2}-\theta_{1}^{*} \theta_{2}^{*} \Theta\right)}{\Theta \theta_{1}^{*} \theta_{2}^{*} \Theta_{1}} \frac{\sigma^{2} \sigma_{\varepsilon}^{2}}{\sigma_{\varepsilon}^{2}+2 \sigma^{2}}, \\
p\left(y_{1}, y_{2}\right) & =\frac{\theta_{1} \theta_{2} \Theta_{1} \Theta_{2}\left(\mu+\left(\frac{y_{1}+y_{2}}{2}-\mu\right) \frac{\sigma^{2}}{\frac{1}{2} \sigma_{\varepsilon}^{2}+\sigma^{2}}\right)}{\theta_{1} \theta_{2} \Theta_{1} \Theta_{2}}-\frac{1}{2} \frac{\theta_{1} \Theta_{2}^{2}+\theta_{2} \Theta_{1}^{2}-\Theta_{1} \theta_{2}}{\theta_{1} \theta_{2} \Theta_{1} \Theta_{2}} \frac{\sigma^{2} \sigma_{\varepsilon}^{2}}{\sigma_{\varepsilon}^{2}+2 \sigma^{2}}
\end{aligned}
$$

is a rational expectations equilibrium with strategic beliefs manipulations.

To sum up, when the agents beliefs result from the observation of signals that are correlated with $\tilde{x}$, the resulting rational expectations equilibrium with strategic beliefs manipulation has

\footnotetext{
${ }^{4}$ Remark that, by construction, we have at the equilibrium $P\left(y_{1}, y_{2}\right)=p\left(\widehat{\mu}_{1}\left(y_{1}, y_{2}\right), \widehat{\mu}_{2}\left(y_{1}, y_{2}\right)\right)$.
} 
the same characteristics as the equilibrium with strategic beliefs manipulation when the agents have the same initial belief $\mathcal{N}\left(\breve{\mu}, \breve{\sigma}^{2}\right)$.

On average over all possible signals, the average beliefs and price correspond to the equilibrium with strategic beliefs manipulation analyzed in the previous section when the distribution of $\tilde{x}$ is assumed to be $\mathcal{N}\left(\mu, \breve{\sigma}^{2}\right)$. Hence, modulo a modification of the variance of $\tilde{x}$, the results provided in Proposition 1 can be seen as corresponding to the average situation in a model with rational expectations and strategic behavior.

\subsection{The model with two risky assets}

The model is essentially the same as in Section 2 except that we now suppose that there are two risky assets in the economy, whose associated payoffs at the end of the period are respectively denoted by $\widetilde{x}$ and $\widetilde{y}$. We let $p$ (resp. $q$ ) denote the price of $\widetilde{x}$ (resp. $\widetilde{y}$ ) and we assume that $\widetilde{x}$ and $\widetilde{y}$ are normally distributed, more precisely $\widetilde{x} \sim \mathcal{N}\left(\mu, \sigma^{2}\right)$ and $\widetilde{y} \sim \mathcal{N}\left(\nu, \varpi^{2}\right)$. We let $\rho$ denote the correlation between $\widetilde{x}$ and $\widetilde{y}$, i.e., $\rho \equiv \frac{\operatorname{cov}(\widetilde{x} \widetilde{y})}{\sigma \varpi}$. We also denote by $\tilde{\xi}^{M} \equiv \widetilde{x}+\widetilde{y}$ the market portfolio payoffs.

We assume that each guru can choose a belief, i.e. a pair $\left(\widehat{\mu}_{i}, \widehat{\nu}_{i}\right)$ that maximizes his utility from trade and as previously we look for an equilibrium with strategic beliefs manipulation. The belief of agent $i$ with respect to the average market portfolio payoff is denoted by $\widehat{\xi}_{i}^{M} \equiv \widehat{\mu}_{i}+\widehat{\nu}_{i}$. The definition and the notations are straightforward generalizations of those introduced in Section 2 .

Proposition 5 1. For given $\Theta_{1}$ and $\Theta_{2}$, there exists a unique equilibrium with strategic beliefs manipulation $\left(\left(\widehat{\mu}_{i}, \widehat{\nu}_{i}\right) ; i=1,2\right)$ where

$$
\begin{aligned}
& \widehat{\mu}_{i}\left(\Theta_{1}, \Theta_{2}\right)=\mu+\frac{1}{2} \frac{\left(\theta_{1}^{*} \Theta_{2}^{2}+\theta_{2}^{*} \Theta_{1}^{2}+2 \theta_{1}^{*} \theta_{2}^{*} \theta_{i}^{*-1} \Theta_{1} \Theta_{2}-\theta_{1}^{*} \theta_{2}^{*} \Theta\right)}{\Theta \theta_{1}^{*} \theta_{2}^{*} \Theta_{1} \Theta_{2}} \Theta_{i}\left(\sigma^{2}+\rho \sigma \varpi\right) \\
& \widehat{\nu}_{i}\left(\Theta_{1}, \Theta_{2}\right)=\nu+\frac{1}{2} \frac{\left(\theta_{1}^{*} \Theta_{2}^{2}+\theta_{2}^{*} \Theta_{1}^{2}+2 \theta_{1}^{*} \theta_{2}^{*} \theta_{i}^{*-1} \Theta_{1} \Theta_{2}-\theta_{1}^{*} \theta_{2}^{*} \Theta\right)}{\Theta \theta_{1}^{*} \theta_{2}^{*} \Theta_{1} \Theta_{2}} \Theta_{i}\left(\varpi^{2}+\rho \sigma \varpi\right)
\end{aligned}
$$

and the associated average market portfolio payoffs $\left(\widehat{\xi}_{i}^{M}\right)_{i=1,2}$ are given by

$$
\widehat{\xi}_{i}^{M}\left(\Theta_{1}, \Theta_{2}\right)=\xi^{M}+\frac{1}{2} \frac{\left(\theta_{1}^{*} \Theta_{2}^{2}+\theta_{2}^{*} \Theta_{1}^{2}+2 \theta_{1}^{*} \theta_{2}^{*} \theta_{i}^{*-1} \Theta_{1} \Theta_{2}-\theta_{1}^{*} \theta_{2}^{*} \Theta\right)}{\Theta \theta_{1}^{*} \theta_{2}^{*} \Theta_{1} \Theta_{2}} \Theta_{i} \sigma_{M}^{2}
$$

where $\sigma_{M}^{2}=\varpi^{2}+2 \rho \sigma \varpi+\sigma^{2}$. 
2. We have

$$
\frac{\widehat{\mu}_{1}-\widehat{\mu}_{2}}{\widehat{\nu}_{1}-\widehat{\nu}_{2}}=\frac{\widehat{\mu}_{1}-\mu}{\widehat{\nu}_{1}-\nu}=\frac{\sigma^{2}+\rho \sigma \varpi}{\varpi^{2}+\rho \sigma \varpi}
$$

and beliefs dispersion is larger for the asset that has higher variance.

3. The equilibrium risk premia for the first and the second asset as well as for the market portfolio are given by

$$
\begin{aligned}
\mu-p & =\frac{1}{2} \frac{\left(\Theta \theta_{1}^{*} \theta_{2}^{*}-\Theta_{1}^{2} \theta_{2}^{*}-\Theta_{2}^{2} \theta_{1}^{*}\right)}{\theta_{2}^{*} \theta_{1}^{*} \Theta_{1} \Theta_{2}}\left(\sigma^{2}+\rho \varpi \sigma\right), \\
\nu-q & =\frac{1}{2} \frac{\left(\Theta \theta_{1}^{*} \theta_{2}^{*}-\Theta_{1}^{2} \theta_{2}^{*}-\Theta_{2}^{2} \theta_{1}^{*}\right)}{\theta_{2}^{*} \theta_{1}^{*} \Theta_{1} \Theta_{2}}\left(\varpi^{2}+\rho \varpi \sigma\right), \\
\xi^{M}-(p+q) & =\frac{1}{2} \frac{\left(\Theta \theta_{1}^{*} \theta_{2}^{*}-\Theta_{1}^{2} \theta_{2}^{*}-\Theta_{2}^{2} \theta_{1}^{*}\right)}{\theta_{2}^{*} \theta_{1}^{*} \Theta_{1} \Theta_{2}} \sigma_{M}^{2} .
\end{aligned}
$$

4. There exists a unique equilibrium $\left(\Theta_{1}^{*}, \Theta_{2}^{*}\right)$ for the groups' dynamics. It is given by Equation (7), as in the one asset framework.

5. If the gurus are on average as risk tolerant as the whole population, i.e. $\Theta=\frac{\theta_{1}^{*}+\theta_{2}^{*}}{2}$, then $\Theta_{1}^{*}>\Theta_{2}^{*}$ and the market risk premium is increased

In fact, in the two assets framework everything works as in a one asset framework where the unique asset is the market portfolio whose average payoff is given by $\xi^{M}=\mu+\nu$ and whose variance is given by $\sigma_{M}^{2}=\varpi^{2}+2 \rho \sigma \varpi+\sigma^{2}$. This means that the more risk tolerant (risk averse) group is pessimistic (resp. optimistic) at the aggregate level and the consensus belief is pessimistic at the aggregate level. The formulas for individual assets that are provided in the proposition are similar to those obtained in the one asset framework. However, for each asset, the variance term in the one-asset formula is replaced by the covariance of the considered asset payoffs with the market portfolio payoffs. Recall that in the Walrasian setting (CAPM setting), the equilibrium price for a given asset depends on the covariance of the payoffs of this asset with the payoffs of the market portfolio and not on the total variance of the asset payoffs. Since the gurus modify their beliefs in order to manipulate the prices, it is natural to obtain optimal beliefs that depend on the covariance with the market portfolio and not on the total variance. The aggregate level properties (pessimism, correlation between pessimism and risk tolerance,...) are then retrieved at the individual assets level as far as these assets are positively correlated with the market portfolio. 
It is interesting to note that these effects are more pronounced for the riskier asset. Intuitively, since the gurus differ by their level of risk tolerance, more risk leads to more beliefs dispersion and hence to a more pronounced impact on the market for the riskier asset.

\subsection{The model with more than 2 gurus}

We assumed so far that there are only 2 gurus in the economy. This is a natural assumption when we only have one asset. Indeed, even if there are more than 2 gurus the condition that all gurus have the same level of prediction accuracy or that all beliefs are at the same distance of the objective belief implies that they will cluster in 2 groups with beliefs that are symmetric with respect to the objective belief: an optimistic one and a pessimistic one. When there are at least 2 assets, the situation is different. Let us illustrate this point in the 2 asset framework: the condition that all gurus have beliefs $\left(\mu_{i}, \nu_{i}\right)$ that are at the same distance of the objective belief $(\mu, \nu)$ only imposes that all the $\left(\mu_{i}, \nu_{i}\right)$ are on a same circle centered on $(\mu, \nu)$.

In the next, we fully analyze the 2 asset and 3 guru framework.

With the same notations as above, solving for the Nash equilibrium leads to

$$
\begin{aligned}
\mu_{i}-\mu & =\frac{1}{2}\left(\frac{\theta_{1} \theta_{2} \Theta_{3}^{2}+\theta_{1} \theta_{3} \Theta_{2}^{2}+\theta_{2} \theta_{3} \Theta_{1}^{2}}{\theta_{1} \theta_{2} \theta_{3} \Theta\left(\Theta_{1} \Theta_{2}+\Theta_{1} \Theta_{3}+\Theta_{2} \Theta_{3}\right)}+\frac{2}{\theta_{i} \Theta}-\frac{1}{\Theta_{1} \Theta_{2}+\Theta_{1} \Theta_{3}+\Theta_{2} \Theta_{3}}\right) \Theta_{i}\left(\sigma^{2}+\rho \sigma \varpi\right), \\
\nu_{i}-\nu & =\frac{1}{2}\left(\frac{\theta_{1} \theta_{2} \Theta_{3}^{2}+\theta_{1} \theta_{3} \Theta_{2}^{2}+\theta_{2} \theta_{3} \Theta_{1}^{2}}{\theta_{1} \theta_{2} \theta_{3} \Theta\left(\Theta_{1} \Theta_{2}+\Theta_{1} \Theta_{3}+\Theta_{2} \Theta_{3}\right)}+\frac{2}{\theta_{i} \Theta}-\frac{1}{\Theta_{1} \Theta_{2}+\Theta_{1} \Theta_{3}+\Theta_{2} \Theta_{3}}\right) \Theta_{i}\left(\varpi^{2}+\rho \sigma \varpi\right) .
\end{aligned}
$$

In particular, for $i=1,2,3$, we have

$$
\frac{\mu_{i}-\mu}{\nu_{i}-\nu}=\frac{\sigma^{2}+\rho \sigma \varpi}{\varpi^{2}+\rho \sigma \varpi}
$$

and all the vectors $\left(\mu_{i}-\mu, \nu_{i}-\nu\right)$ are then proportional. Imposing that all the gurus have the same level of prediction accuracy (or that all beliefs are at the same distance of the objective beliefs) leads then to a situation where at least two gurus have exactly the same beliefs. Therefore, the 3 guru framework can be reduced to a 2 gurus framework. The same result can be obtained for $N$ gurus. 


\section{Conclusion}

When market opinions are driven by gurus and when these gurus act strategically, this leads to heterogeneous and antagonistic beliefs. The agents cluster in two groups (even if there are more than two gurus). The beliefs of the more risk averse group exhibit optimism while the beliefs of the more risk tolerant group exhibit pessimism. As a consequence, there is a positive correlation between pessimism and risk tolerance and the representative agent belief exhibits pessimism. This induced pessimism of the representative agent is interesting in light of the risk premium puzzle. It is interesting to notice that the different effects we exhibit (beliefs heterogeneity, representative agent pessimism, impact on the risk premium) are more pronounced for riskier assets.

This work suggests further investigation. It would be useful to analyze how our results can be transposed in a dynamic setting where agents then have dynamic strategies. We focused on a steady state but it would be interesting to analyze how the gurus dynamically interact, how their reputation evolves through time and how they converge to the steady state.

\section{Appendix Proofs}

\section{Proof of Proposition 1}

Guru 1 choses the belief $\widehat{\mu}_{1}$ that maximizes

$$
A_{1}\left(\mu_{1}\right)=\theta_{1} \frac{\mu_{1}-p\left(\mu_{1}, \widehat{\mu}_{2}\right)}{\sigma^{2}}\left(\mu-p\left(\mu_{1}, \widehat{\mu}_{2}\right)\right)+p\left(\mu_{1}, \widehat{\mu}_{2}\right)-\frac{1}{2} \frac{1}{\theta_{1}}\left[\theta_{1} \frac{\mu_{1}-p\left(\mu_{1}, \widehat{\mu}_{2}\right)}{\sigma^{2}}\right]^{2} \sigma^{2}
$$

where $p\left(\mu_{1}, \mu_{2}\right)=\frac{\Theta_{1} \mu_{1}+\Theta_{2} \mu_{2}}{\Theta_{1}+\Theta_{2}}-\frac{\sigma^{2}}{\Theta_{1}+\Theta_{2}}$ and where $\widehat{\mu}_{2}$ is considered as given. Symmetrically, Guru 2 choses the belief $\widehat{\mu}_{1}$ that maximizes

$$
A_{2}\left(\mu_{2}\right)=\theta_{2} \frac{\mu_{2}-p\left(\widehat{\mu}_{1}, \mu_{2}\right)}{\sigma^{2}}\left(\mu-p\left(\widehat{\mu}_{1}, \mu_{2}\right)\right)+\frac{1}{2} p\left(\widehat{\mu}_{1}, \mu_{2}\right)-\frac{1}{2} \frac{1}{\theta_{2}}\left[\theta_{2} \frac{\mu_{2}-p\left(\widehat{\mu}_{1}, \mu_{2}\right)}{\sigma^{2}}\right]^{2} \sigma^{2}
$$

where $\widehat{\mu}_{1}$ is considered as given. Solving for $\frac{d A_{1}}{d \mu_{1}}\left(\widehat{\mu}_{1}, \widehat{\mu}_{2}\right)=\frac{d A_{2}}{d \mu_{2}}\left(\widehat{\mu}_{1}, \widehat{\mu}_{2}\right)=0$ leads to Equations (5) and (6).

\section{Proof of Proposition 2}

1. Let us assume that $\left|\mu_{1}-\mu\right|=\left|\mu-\mu_{2}\right|$. This leads to $\mu_{1}=\mu_{2}$ or to $\mu_{1}-\mu=\mu-\mu_{2}$.

There is no solution associated to $\mu_{1}=\mu_{2}$. Indeed, using Equations (5) and (6), the condition 
$\mu_{1}=\mu_{2}$ leads to $\Theta_{2} \theta_{1}^{*} \theta_{2}^{*}-\Theta_{1} \theta_{1}^{*} \theta_{2}^{*}+\Theta_{1}^{2} \theta_{2}^{*}-\Theta_{2}^{2} \theta_{1}^{*}=0$ or equivalently to $\Theta_{1}^{2}\left(\theta_{2}^{*}-\theta_{1}^{*}\right)+\Theta_{1}$ $\left(2 \Theta \theta_{1}^{*}-2 \theta_{1}^{*} \theta_{2}^{*}\right)+\Theta \theta_{1}^{*} \theta_{2}^{*}-\Theta^{2} \theta_{1}^{*}=0$. The discriminant associated to this polynomial equation in $\Theta_{1}^{2}$ is given by $4 \theta_{1}^{*} \theta_{2}^{*}\left(\theta_{2}^{*}-\Theta\right)\left(\theta_{1}^{*}-\Theta\right)$ which is negative.

Using Equations (5) and (6), the condition $\mu_{1}-\mu=\mu-\mu_{2}$ is equivalent to

$$
H\left(\Theta_{1}\right) \equiv 2 \Theta_{1}^{3}\left(\theta_{1}^{*}-\theta_{2}^{*}\right)-3 \Theta \Theta_{1}^{2}\left(\theta_{1}^{*}-\theta_{2}^{*}\right)+\Theta^{2} \theta_{1}^{*}\left(\Theta-\theta_{2}^{*}\right)=0
$$

We have $H(0)=\Theta^{2} \theta_{1}^{*}\left(\Theta-\theta_{2}^{*}\right)>0$ and $H(\Theta)=\Theta^{2} \theta_{2}^{*}\left(\Theta-\theta_{1}^{*}\right)<0$. It is easy to check that $H$ is decreasing on $(0, \Theta)$ since $H^{\prime}\left(\Theta_{1}\right)=6 \Theta_{1}\left(\Theta_{1}-\Theta\right)\left(\theta_{1}^{*}-\theta_{2}^{*}\right)$ is negative on this interval. The equation $H\left(\Theta_{1}\right)=0$ admits then only one solution in $(0, \Theta)$.

2. In order analyze the stability of the equilibrium, let us analyze the signs of $\mu_{1}-\mu, \mu_{2}-\mu$ and $\mu_{1}+\mu_{2}-2 \mu$. It is easy to prove that the sign of $\mu_{1}+\mu_{2}-2 \mu$ is the same as the sign of $H\left(\Theta_{1}\right)$, the sign of $\mu_{1}-\mu$ is the same as the sign of $G\left(\Theta_{1}\right)=\Theta_{1}^{2}\left(\theta_{1}^{*}-\theta_{2}^{*}\right)-2 \Theta \Theta_{1}\left(\theta_{1}^{*}-\theta_{2}^{*}\right)+\Theta \theta_{1}^{*}\left(\Theta-\theta_{2}^{*}\right)$ and the sign of $\mu_{2}-\mu$ is the same as the sign of $K\left(\Theta_{1}\right)=-\Theta_{1}^{2}\left(\theta_{1}^{*}-\theta_{2}^{*}\right)+\Theta \theta_{1}^{*}\left(\Theta-\theta_{2}^{*}\right)$. First, under the assumption $\theta_{1}^{*}>\Theta>\theta_{2}^{*}$, we already proved that $H(0)>0$ and $H(\Theta)<0$ and that $H$ admits only one root on $[0, \Theta]$. Let us denote by $\Theta^{H}$ this root. Second, we prove that $G$ has only one root on $[0, \Theta]$. Indeed, the sum of its roots is equal to $2 \Theta$ and we have $G(0)>0$ and $G(\Theta)<0$. Let us denote by $\Theta^{G}$ this root. Third, we have $K(0)>0$ and $K(\Theta)<0$ and it is easy to see that $K$ has a unique root on $[0, \Theta]$. Let us denote by $\Theta^{K}$ this root. We can easily check that $H\left(\Theta^{G}\right)>0$ which gives us $\Theta^{G}<\Theta^{H}$. With similar arguments we can prove that $\Theta^{H}<\Theta^{K}$. Finally, we can easily show that we always have $\mu_{2}>\mu_{1}$.

We sum up these results in the following table where $\breve{\mu}_{i}$ is the symmetric of $\mu_{i}$ with respect to $\mu$, i.e. $\breve{\mu}_{i}=2 \mu-\mu_{i}$.

\begin{tabular}{|l||l|l|l|l|}
\hline$\Theta$ & $\left(0, \Theta^{G}\right)$ & $\left(\Theta^{G}, \Theta^{H}\right)$ & $\left(\Theta^{H}, \Theta^{K}\right)$ & $\left(\Theta^{K}, \Theta\right)$ \\
\hline \hline$G$ & $\mu_{1}>\mu$ & $\mu_{1}<\mu$ & $\mu_{1}<\mu$ & $\mu_{1}<\mu$ \\
\hline$H$ & $\mu_{1}+\mu_{2}>2 \mu$ & $\mu_{1}+\mu_{2}>2 \mu$ & $\mu_{1}+\mu_{2}<2 \mu$ & $\mu_{1}+\mu_{2}<2 \mu$ \\
\hline$K$ & $\mu_{2}>\mu$ & $\mu_{2}>\mu$ & $\mu_{2}>\mu$ & $\mu_{2}<\mu$ \\
\hline$\left(\mu, \mu_{1}, \mu_{2}\right)$ & $\mu<\mu_{1}<\mu_{2}$ & $\mu_{1}<\mu<\breve{\mu}_{1}<\mu_{2}$ & $\mu_{1}<\breve{\mu}_{2}<\mu<\mu_{2}$ & $\mu_{1}<\mu_{2}<\mu$ \\
\hline$\dot{\Theta}_{1}$ & $\dot{\Theta}_{1}>0$ & $\dot{\Theta}_{1}>0$ & $\dot{\Theta}_{1}<0$ & $\dot{\Theta}_{1}<0$ \\
\hline
\end{tabular}

By definition, we have $\Theta_{1}^{*}=\Theta^{H}$. Consequently, we have $\dot{\Theta}_{1}>0$ for $\Theta_{1}<\Theta_{1}^{*}$ and $\dot{\Theta}_{1}<0$ for $\Theta_{1}>\Theta_{1}^{*}$. 
3. Let us consider the utility $U_{\theta}^{i}$ of an agent with risk tolerance level $\theta$ that follows Guru $i$ and let us denote by $j \neq i$ the other guru. We have

$$
\begin{aligned}
U_{\theta}^{i} & =\exp \frac{1}{\theta}\left(p\left(\widehat{\mu}_{1}, \widehat{\mu}_{2}\right)+\theta \frac{\mu_{i}-p\left(\widehat{\mu}_{1}, \widehat{\mu}_{2}\right)}{\sigma^{2}}\left(\mu-p\left(\widehat{\mu}_{1}, \widehat{\mu}_{2}\right)\right)-\frac{1}{2} \frac{1}{\theta}\left(\theta \frac{\mu_{i}-p\left(\widehat{\mu}_{1}, \widehat{\mu}_{2}\right)}{\sigma^{2}}\right)^{2} \sigma^{2}\right) \\
& =\exp \left(\frac{p\left(\widehat{\mu}_{1}, \widehat{\mu}_{2}\right)}{\theta}+\frac{\mu_{i}-p\left(\widehat{\mu}_{1}, \widehat{\mu}_{2}\right)}{\sigma^{2}}\left(\frac{1}{2} \mu-\frac{1}{2} \mu_{i}-\frac{1}{2} p\left(\widehat{\mu}_{1}, \widehat{\mu}_{2}\right)\right)\right) \\
& =\exp \left(\frac{p\left(\widehat{\mu}_{1}, \widehat{\mu}_{2}\right)}{\theta}+\frac{\mu_{i}-p\left(\widehat{\mu}_{1}, \widehat{\mu}_{2}\right)}{\sigma^{2}}\left(\frac{\mu_{1}+\mu_{2}}{2}-\frac{1}{2} \mu_{i}-\frac{1}{2} p\left(\widehat{\mu}_{1}, \widehat{\mu}_{2}\right)\right)\right) \\
& =\exp \left(\frac{p\left(\widehat{\mu}_{1}, \widehat{\mu}_{2}\right)}{\theta}+\frac{1}{2 \sigma^{2}}\left(\mu_{i}-p\left(\widehat{\mu}_{1}, \widehat{\mu}_{2}\right)\right)\left(\mu_{j}-p\left(\widehat{\mu}_{1}, \widehat{\mu}_{2}\right)\right)\right) .
\end{aligned}
$$

We then have $U_{\theta}^{i}=U_{\theta}^{j}$ and the utility level does not depend on the choice of a specific guru.

4. Let us now assume that $\Theta=\frac{\theta_{1}^{*}+\theta_{2}^{*}}{2}$. Under this assumption we obtain $H\left(\frac{\theta_{1}^{*}+\theta_{2}^{*}}{4}\right)<0$ and $H\left(\frac{\theta_{1}^{*}+\theta_{2}^{*}}{2}\right)>0$ which implies that $\Theta_{1}^{*} \in\left(\frac{\theta_{1}^{*}+\theta_{2}^{*}}{4}, \frac{\theta_{1}^{*}+\theta_{2}^{*}}{2}\right)$ and $\Theta_{2}^{*} \in\left(0, \frac{\theta_{1}^{*}+\theta_{2}^{*}}{4}\right)$. In particular, we have $\Theta_{1}^{*}>\Theta_{2}^{*}$. Let us prove that Group 1 is more pessimistic than Group 2 or equivalently that $\widehat{\mu}_{1}-\mu<0$. We have then to prove that $G\left(\Theta^{H}\right)<0$. This results directly from the table above. Group 1 and Guru 1 are then more pessimistic then Group 2 and Guru 2. Pessimism and risk tolerance are then positively correlated at the gurus level.

5. If the two groups are of equal size, the average belief is then the objective belief. The average risk tolerance in Group 1 (resp. Group 2) is then equal to $2 \Theta_{1}^{*}$ (resp. 2 $2 \Theta_{2}^{*}$ ) and the covariance between optimism and risk tolerance is then given by $\left(\Theta_{1}^{*}-\Theta_{2}^{*}\right)\left(\widehat{\mu}_{1}-\mu\right)<0$.

Proof of Proposition 3 When Guru 1 has an initial belief $\nu_{1}$ and Guru 2 has an initial belief $\nu_{2}$, they announce beliefs $\left(\widehat{\mu}_{1}, \widehat{\mu}_{2}\right)$ such that $\widehat{\mu}_{1}$ maximizes

$$
A_{1}\left(\mu_{1}, \widehat{\mu}_{2}\right)=\theta_{1} \frac{\mu_{1}-p\left(\mu_{1}, \widehat{\mu}_{2}\right)}{\sigma^{2}}\left(\nu_{1}-p\left(\mu_{1}, \widehat{\mu}_{2}\right)\right)+p\left(\mu_{1}, \widehat{\mu}_{2}\right)-\frac{1}{2} \frac{1}{\theta_{1}}\left[\theta_{1} \frac{\mu_{1}-p\left(\mu_{1}, \widehat{\mu}_{2}\right)}{\sigma^{2}}\right]^{2} \sigma^{2}
$$

the belief $\widehat{\mu}_{2}$ being given and such that $\widehat{\mu}_{2}$ maximizes

$$
A_{2}\left(\widehat{\mu}_{1}, \mu_{2}\right)=\theta_{2} \frac{\mu_{2}-p\left(\widehat{\mu}_{1}, \mu_{2}\right)}{\sigma^{2}}\left(\nu_{2}-p\left(\widehat{\mu}_{1}, \mu_{2}\right)\right)+p\left(\widehat{\mu}_{1}, \mu_{2}\right)-\frac{1}{2} \frac{1}{\theta_{2}}\left[\theta_{2} \frac{\mu_{2}-p\left(\widehat{\mu}_{1}, \mu_{2}\right)}{\sigma^{2}}\right]^{2} \sigma^{2}
$$

the belief $\widehat{\mu}_{1}$ being given and where $p\left(\mu_{1}, \mu_{2}\right)=\frac{\Theta_{1} \mu_{1}+\Theta_{2} \mu_{2}}{\Theta}-\frac{\sigma^{2}}{\Theta}$ (market clearing condition). The first order conditions $\frac{d A_{1}}{d \mu_{1}}\left(\widehat{\mu}_{1}, \widehat{\mu}_{2}\right)=\frac{d A_{2}}{d \mu_{2}}\left(\widehat{\mu}_{1}, \widehat{\mu}_{2}\right)=0$ can be rewritten as follows 


$$
\begin{aligned}
& \widehat{\mu}_{1}=\frac{\theta_{1} \Theta_{1} \Theta_{2} \widehat{\mu}_{2}+\theta_{1} \Theta_{1} \Theta_{2} \nu_{1}+\sigma^{2} \Theta_{1} \Theta_{2}+\theta_{1} \Theta_{2}^{2} \nu_{1}+\sigma^{2} \Theta_{1}^{2}-\sigma^{2} \theta_{1} \Theta_{1}}{2 \theta_{1} \Theta_{1} \Theta_{2}+\theta_{1} \Theta_{2}^{2}} \\
& \widehat{\mu}_{2}=\frac{\theta_{2} \Theta_{1} \Theta_{2} \widehat{\mu}_{1}+\theta_{2} \Theta_{1} \Theta_{2} \nu_{2}-\sigma^{2} \theta_{2} \Theta_{2}+\sigma^{2} \Theta_{1} \Theta_{2}+\theta_{2} \Theta_{1}^{2} \nu_{2}+\sigma^{2} \Theta_{2}^{2}}{2 \theta_{2} \Theta_{1} \Theta_{2}+\theta_{2} \Theta_{1}^{2}}
\end{aligned}
$$

and solving this system for $\left(\widehat{\mu}_{1}, \widehat{\mu}_{2}\right)$ gives the result.

Proof of Proposition 4 It suffices to check that $\left(\left(\widehat{\mu}_{1}\left(y_{1}, y_{2}\right), \widehat{\mu}_{2}\left(y_{1}, y_{2}\right), P\left(y_{1}, y_{2}\right)\right)\right.$ is a rational expectation equilibrium with strategic beliefs manipulation. Remark that $P\left(y_{1}, y_{2}\right)$ satisfes the condition $P\left(y_{1}, y_{2}\right)=\frac{\Theta_{1} \widehat{\mu}_{1}\left(y_{1}, y_{2}\right)+\Theta_{2} \widehat{\mu}_{1}\left(y_{1}, y_{2}\right)}{\Theta}-\frac{\sigma^{2}}{\Theta}$ and $P\left(y_{1}, y_{2}\right)$ is then the equilibrium price when Guru 1 announces $\widehat{\mu}_{1}\left(y_{1}, y_{2}\right)$ and Guru 2 announces $\widehat{\mu}_{2}\left(y_{1}, y_{2}\right)$. Since $P\left(y_{1}, y_{2}\right)$ reveals $\frac{y_{1}+y_{2}}{2}$ and since $\frac{y_{1}+y_{2}}{2}$ is a sufficient statistics of all available information, everything works as if each agent would take expectations that are conditional to $\frac{y_{1}+y_{2}}{2}$. They would share then the same belief $\mathcal{N}\left(\breve{\mu}, \breve{\sigma}^{2}\right)$ with $\breve{\mu}=\mu+(\bar{y}-\mu) \frac{\sigma^{2}}{\frac{1}{2} \sigma_{\varepsilon}^{2}+\sigma^{2}}$ and $\breve{\sigma}^{2}=\frac{\sigma^{2} \sigma_{\varepsilon}^{2}}{\sigma_{\varepsilon}^{2}+2 \sigma^{2}}$. Their announced beliefs would then be determined by Equations (5) and (6) replacing $\left(\mu, \sigma^{2}\right)$ by $\left(\breve{\mu}, \breve{\sigma}^{2}\right)$.

Proof of Proposition 5 When there are 2 assets and when Guru 1 announces $\left(\mu_{1}, \nu_{1}\right)$ and Guru 2 announces $\left(\mu_{2}, \nu_{2}\right)$ the optimal demands of Group $i$ in asset 1 and in asset 2 are respectively given by

$$
\begin{aligned}
\alpha_{G_{i}} & =\Theta_{i} \frac{\mu_{i}-p}{\sigma^{2}\left(1-\rho^{2}\right)}-\Theta_{i} \frac{\left(\nu_{i}-q\right) \rho}{\sigma \varpi\left(1-\rho^{2}\right)} \\
\beta_{G_{i}} & =\Theta_{i} \frac{\nu_{i}-q}{\varpi^{2}\left(1-\rho^{2}\right)}-\Theta_{i} \frac{\left(\mu_{i}-p\right) \rho}{\sigma \varpi\left(1-\rho^{2}\right)}
\end{aligned}
$$

and by the market clearing condition, the equilibrium prices are given by

$$
p=\frac{\Theta_{1} \mu_{1}+\Theta_{2} \mu_{2}}{\Theta}-\frac{\sigma^{2}+\sigma \varpi \rho}{\Theta}, \quad q=\frac{\Theta_{1} \nu_{1}+\Theta_{2} \nu_{2}}{\Theta}-\frac{\varpi^{2}+\sigma \varpi \rho}{\Theta} .
$$

The optimal demands $\alpha_{i}$ and $\beta_{i}$ of Guru $i$ in asset 1 and in asset 2 are respectively given by

$$
\begin{aligned}
& \alpha_{i}=\theta_{i}^{*} \frac{\mu_{1}-p}{\sigma^{2}\left(1-\rho^{2}\right)}-\theta_{i}^{*} \frac{\left(\nu_{1}-q\right) \rho}{\sigma \varpi\left(1-\rho^{2}\right)} \\
& \beta_{i}=\theta_{i}^{*} \frac{\nu_{1}-q}{\varpi^{2}\left(1-\rho^{2}\right)}-\theta_{i}^{*} \frac{\left(\mu_{1}-p\right) \rho}{\sigma \varpi\left(1-\rho^{2}\right)}
\end{aligned}
$$


Guru $i$ maximizes then

$$
A_{i}=p+\alpha_{i}(\mu-p)+q+\beta_{i}(\nu-q)-\frac{1}{2} \frac{1}{\theta_{i}^{*}}\left(\alpha_{i}^{2} \sigma^{2}+\beta_{i}^{2} \varpi^{2}+2 \alpha_{i} \beta_{i} \sigma \varpi \rho\right)
$$

with respect to $\left(\mu_{i}, \nu_{i}\right)$ the belief of the other guru being given. The first order conditions $\frac{d A_{1}}{d \mu_{1}}=\frac{d A_{1}}{d \nu_{1}}=\frac{d A_{2}}{d \mu_{2}}=\frac{d A_{2}}{d \nu_{2}}=0$ can be rewritten as follows

$$
\begin{aligned}
\mu_{1} & =\frac{\sigma \varpi \rho \Theta_{1} \Theta_{2}-\sigma \varpi \rho \theta_{1} \Theta_{1}+\mu \theta_{1} \Theta_{1} \Theta_{2}+\theta_{1} \Theta_{1} \Theta_{2} \mu_{2}+\sigma \varpi \rho \Theta_{1}^{2}-\sigma^{2} \theta_{1} \Theta_{1}+\mu \theta_{1} \Theta_{2}^{2}+\sigma^{2} \Theta_{1} \Theta_{2}+\sigma^{2} \Theta_{1}^{2}}{\left(2 \Theta_{1}+\Theta_{2}\right) \Theta_{2} \theta_{1}}, \\
\nu_{1} & =\frac{\sigma \varpi \rho \Theta_{1} \Theta_{2}-\sigma \varpi \rho \theta_{1} \Theta_{1}+\nu \theta_{1} \Theta_{1} \Theta_{2}+\theta_{1} \Theta_{1} \Theta_{2} \nu_{2}+\sigma \varpi \rho \Theta_{1}^{2}-\varpi^{2} \theta_{1} \Theta_{1}+\varpi^{2} \Theta_{1} \Theta_{2}+\nu \theta_{1} \Theta_{2}^{2}+\varpi^{2} \Theta_{1}^{2}}{\left(2 \Theta_{1}+\Theta_{2}\right) \Theta_{2} \theta_{1}}, \\
\mu_{2} & =\frac{\sigma \varpi \rho \Theta_{1} \Theta_{2}-\sigma \varpi \rho \theta_{2} \Theta_{2}+\mu \theta_{2} \Theta_{1} \Theta_{2}+\theta_{2} \Theta_{1} \Theta_{2} \mu_{1}+\sigma \varpi \rho \Theta_{2}^{2}-\sigma^{2} \theta_{2} \Theta_{2}+\mu \theta_{2} \Theta_{1}^{2}+\sigma^{2} \Theta_{1} \Theta_{2}+\sigma^{2} \Theta_{2}^{2}}{\left(2 \Theta_{2}+\Theta_{1}\right) \Theta_{1} \theta_{2}}, \\
\nu_{2} & =\frac{\sigma \varpi \rho \Theta_{1} \Theta_{2}-\sigma \varpi \rho \theta_{2} \Theta_{2}+\nu \theta_{2} \Theta_{1} \Theta_{2}+\theta_{2} \Theta_{1} \Theta_{2} \nu_{1}+\sigma \varpi \rho \Theta_{2}^{2}-\varpi^{2} \theta_{2} \Theta_{2}+\varpi^{2} \Theta_{1} \Theta_{2}+\nu \theta_{2} \Theta_{1}^{2}+\varpi^{2} \Theta_{2}^{2}}{\left(2 \Theta_{2}+\Theta_{1}\right) \Theta_{1} \theta_{2}} .
\end{aligned}
$$

Solving for $\left(\mu_{i}, \nu_{i}\right)$ gives 1 . and 2. and 3 .

Since we have $\frac{\mu_{1}-\mu}{\nu_{1}-\nu}=\frac{\mu_{2}-\mu}{\nu_{2}-\nu}$, the condition $\left(\mu_{1}-\mu\right)^{2}+\left(\nu_{1}-\nu\right)^{2}=\left(\mu_{2}-\mu\right)^{2}+\left(\nu_{2}-\nu\right)^{2}$ can be rewritten as follows

$$
\left|\mu_{1}-\mu\right|=\left|\mu_{2}-\mu\right|
$$

leading to the same equations as in Proposition 2. This proves 4. and 5.

\section{References}

[1] Abel, A., 1989. Asset prices under heterogeneous beliefs: implications for the equity premium. Mimeo, University of Pennsylvania.

[2] Anderson, M. F., A de Palma and J.-F. Thisse, 1992. Discrete choice theory of product differentiation, MIP Press, Cambridge.

[3] Ben Mansour, S., Jouini, E. and C. Napp, 2006. Is There a "Pessimistic" Bias in Individual Beliefs? Evidence from a Simple Survey. Theory and Decision, 61, 345-362.

[4] Benabou, R. and G. Laroque, 1992, Using Privileged Information to Manipulate Markets: Insiders, Gurus, and Credibility, The Quarterly Journal of Economics, 107, 921-958.

[5] Brock, W., and S. Durlauf, 2001a. Discrete choice with social interactions. The Review of Economic Studies, 68, 235-260. 
[6] Brock, W., and S. Durlauf, 2001b. Interaction-based models, in J. Heckman and E. Leamer (Eds), Handbook of Econometrics, vol. V, North-Holland, Amsterdam.

[7] Calvet, L., Grandmont, J.-M. and I. Lemaire, 2002. Aggregation of heterogeneous beliefs and asset pricing in complete financial markets. Working Paper.

[8] Detemple, J. and S. Murthy, 1994. Intertemporal asset pricing with heterogeneous beliefs. Journal of Economic Theory, 62, 294-320.

[9] Fisher, K. and M. Statman, 2000. Investor Sentiment and Stock Returns. Financial Analysts Journal 56, 16-23.

[10] Föllmer, H., U. Horst and A. Kirman, 2004. Equilibria in Financial Markets with Heterogeneous Agents: A Probabilistic Perspective. Journal of Mathematical Economics 41,123-155.

[11] Giordani, P. and P. Söderlind, 2006. Is there evidence of pessimism and doubt in subjective distributions? Implications for the risk premium puzzle. Journal of Economic Dynamics and Control, 30, 1027-1043

[12] Gollier, C., 2007. Who should we believe ? Collective risk taking with heterogeneous beliefs. Journal of Risk and Uncertainty, 35, 107-127.

[13] Jouini, E. and C. Napp, 2006. Heterogeneous beliefs and asset pricing in discrete time: An analysis of pessimism and doubt. Journal of Economic Dynamics and Control 30, 12331260.

[14] Jouini, E., Napp, C. and Y. Viossat, 2010. Evolutionary strategic beliefs. Working Paper.

[15] Lintner, J., 1965. The valuation of risky assets and the selection of risky investments in stock portfolios and capital budgets. Review of Economics and Statistics, 47: 13-37.

[16] Sharpe,W.F., 1964. Capital Asset Prices: A Theory of Market Equilibrium Under Conditions of Risk. Journal of Finance, 19, 425-442.

[17] Shefrin, H., 2005. A Behavioral Approach to Asset Pricing, Academic Press, Advanced Finance Series.

[18] Wakker, P., 2001. Testing and Characterizing Properties of Nonadditive Measures through Violations of the Sure-Thing Principle. Econometrica 69 (4), 1039-1059. 
[19] Weisbuch, G., O. Chenevez, A. Kirman and J. P. Nadal, 1998. A formal approach to market organization: choice functions, mean field approximations and maximum entropy principle, in Advances in Self-Organization and Evolutionary Economics, J. Lesourne and A. Orléan (Eds), Economica, 149-159. 